\title{
The development of an intelligent surveillance system for Korea urban transit
}

\author{
K.-M. Lee, J.-H. Baek, T.-K. Ahn, G.-O. Kim \& J.-H. Lee \\ Train Control and Communication Research Team, \\ Korea Railroad Research Institute (KRRI), Korea
}

\begin{abstract}
Korea Railroad Research Institute (KRRI) is developing an intelligent surveillance system which enables rapid grasping of situations and taking measures by preventing various accidents within the operation section and by supervising urgent and dangerous conditions intelligently when any accident and disaster occurred in order to provide passive services within the section of existing urban transit actively. Intelligent surveillance system analyzes the location and pattern of things by itself by digitalizing imaging information of surveillance camera and enables vulnerability occurred due to the management by person to be replaced and supplemented by operating alarm and access blocking systems or by informing current situations to the security personnel immediately in case where any dangerous situation is occurred. Although there already exists operation issues, such as the legal problems in accordance with the infringement of personal privacy and technical issues like high-resolution camera and broadband transmission media, and the configuration of management scenario which must be built in consideration of violations possible to be occurred in advance to build intelligent surveillance systems in Korea, it is forecast that the demand to build intelligent surveillance systems will be much higher in the future due to the good materials such as the request for a nextgeneration railway management system, growing operational costs in accordance with the increase in labor costs for security, promotion of the revision to laws to make the image of a surveillance camera beneficial as legal evidence, raising the perception of the security industry and customers.
\end{abstract}

Keywords: intelligent surveillance system, urban transit. 


\section{Introduction}

Intelligent surveillance system performs functions such as the fire detection and sensing of structural soundness, etc. within the station building of urban transit through interlocking with sensor on the basis of intelligent surveillance camera. The intelligent image network camera is the independent intelligent video analysis and processing equipment, and it is the equipment which receives camera images and outputs data for result of analysis together with images and makes the alarm occurred. It analyzes violated acts, etc. in accordance with that rule after receiving an analytics rule through the manager program which generates the analytics rule that is the standard for video analysis on a realtime basis [3]. And it outputs the result together with images in the form of meta data [4].

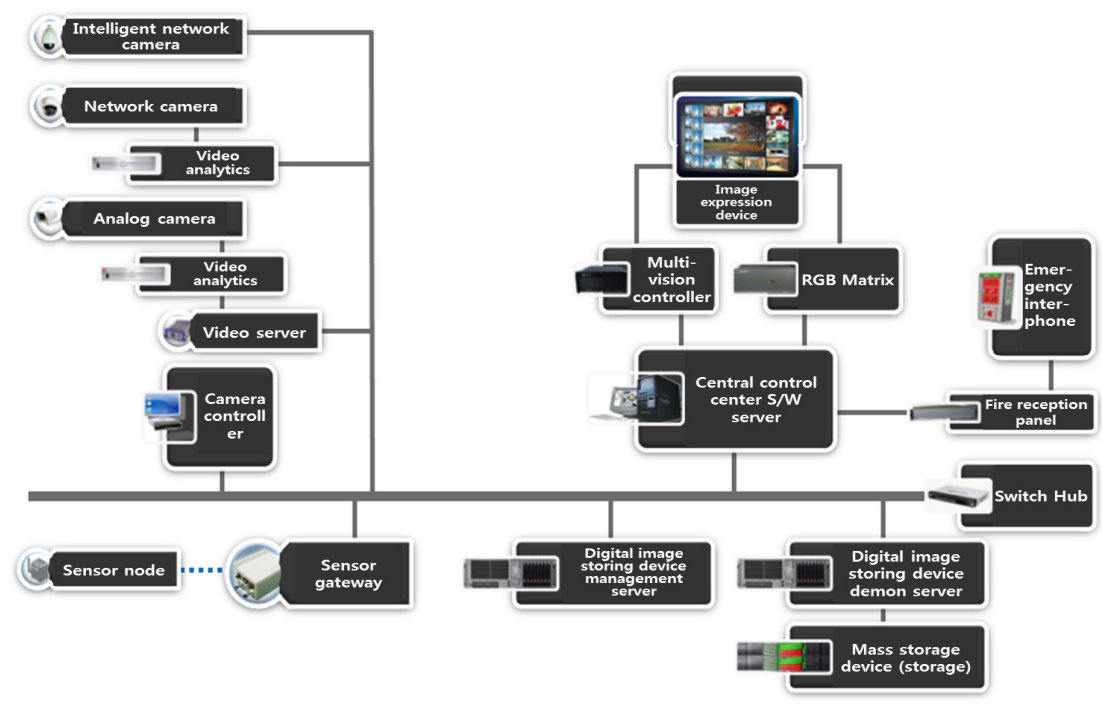

Figure 1: Configuration chart of intelligent surveillance system.

The intelligent surveillance system being developed by the Korea Railroad Research Institute (KRRI) is divided into the intelligent surveillance camera, intelligent sensor network and intelligent central control center surveillance software, and it converts and compresses analog images to digital images by developing the intelligent video analytics, video server to interface with existing cameras other than the intelligent surveillance camera, and transmits event contents through digital video analysis to the intelligent central control center surveillance software through the network. And in the intelligent sensor network, it develops the gateway which integrates data by interfacing existing sensors with new sensors, and transmits it to the intelligent central control center surveillance software through the network. 
The intelligent central control center surveillance software synthesizes and analyzes digital images of intelligent surveillance camera and events, and the sensor data coming through the intelligent sensor network, and all of the data coming into it by linking the fire surveillance panel and failure reception panel which are existing systems, and it expresses its synthetic judgment on situation according to it and contents of event by function on the screen, and plays the role of storing and searching all of the digital images and contents of event [5].

In case where the system is consisted on the basis of this configuration, the configuration chart of intelligent surveillance system to be installed at the new station building or branch office of management is as below. The intelligent network camera can be interlinked with CMS and sensors directly without any separate equipment [6], and it is possible to be built as an intelligent system by using the video analytics and video server to utilize existing systems.

\section{Derivation of the function for intelligent surveillance system}

The intelligent surveillance system is developed to have following functions in the section of urban transit operation.

\subsection{Invasion detection function in the restricted area}

The invasion surveillance function in the restricted area is the function to receive event signals to be transmitted from the intelligent surveillance camera and sensor and to express them on the display device, and to make the surveillance operator recognized by transmitting them to the central control center.

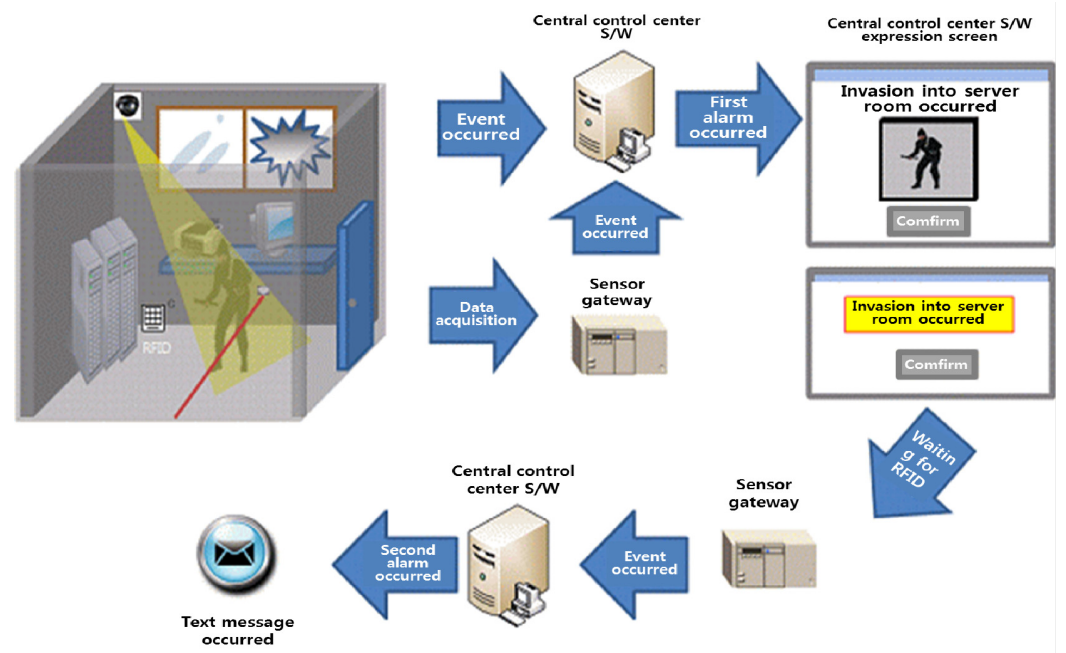

Figure 2: $\quad$ Invasion surveillance scenario in the restricted area. 


\subsection{Passenger flow/degree of crowdedness surveillance function}

Surveillance on the passenger flow and degree of crowdedness measurement is the function to measure and supervise at the intelligent surveillance camera, and to store them in the database by transmitting measured data to the intelligent surveillance system in a fixed cycle, and to transmit and express event logs when the passenger flow and degree of crowdedness are occurred in excess of those designated by the user.

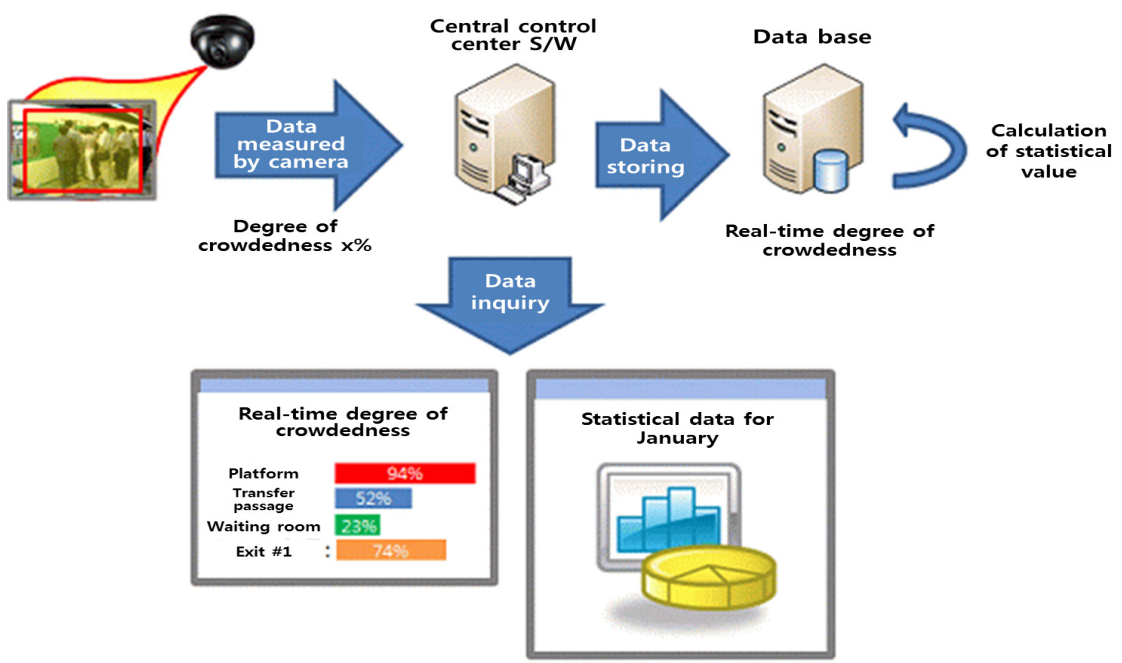

Figure 3: $\quad$ Passenger flow/degree of crowdedness surveillance scenario.

\subsection{Designated/crime-ridden area surveillance function}

Designated/crime-ridden area surveillance and abnormal actor surveillance function is the surveillance function by the user to the actor who stayed or wandered about for more than a fixed time at a specific location or area, and it expresses data on the display device if the intelligent surveillance camera transmits event signals and make the operator recognized by transmitting events to the central control center.

\subsection{Station building structural soundness surveillance function}

Station building structural soundness surveillance is the function to supervise structural safety of the station building from the works, earthquake, etc. around that station building by receiving the information analyzed at the algorithm for structural soundness of station building on the basis of measured value which was measured at the sensor. 


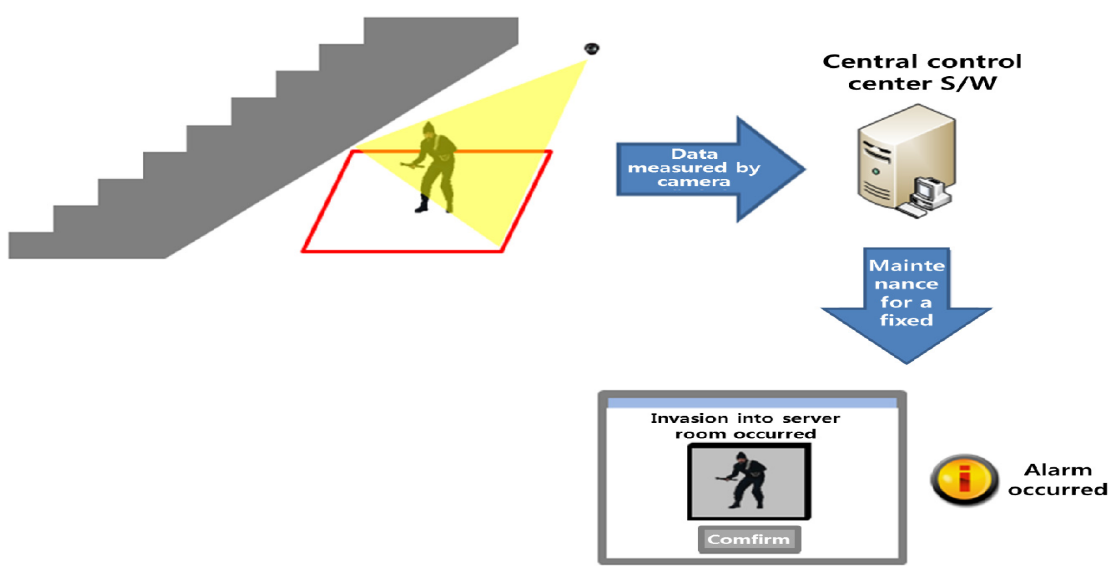

Figure 4: Designated/crime-ridden area surveillance scenario.

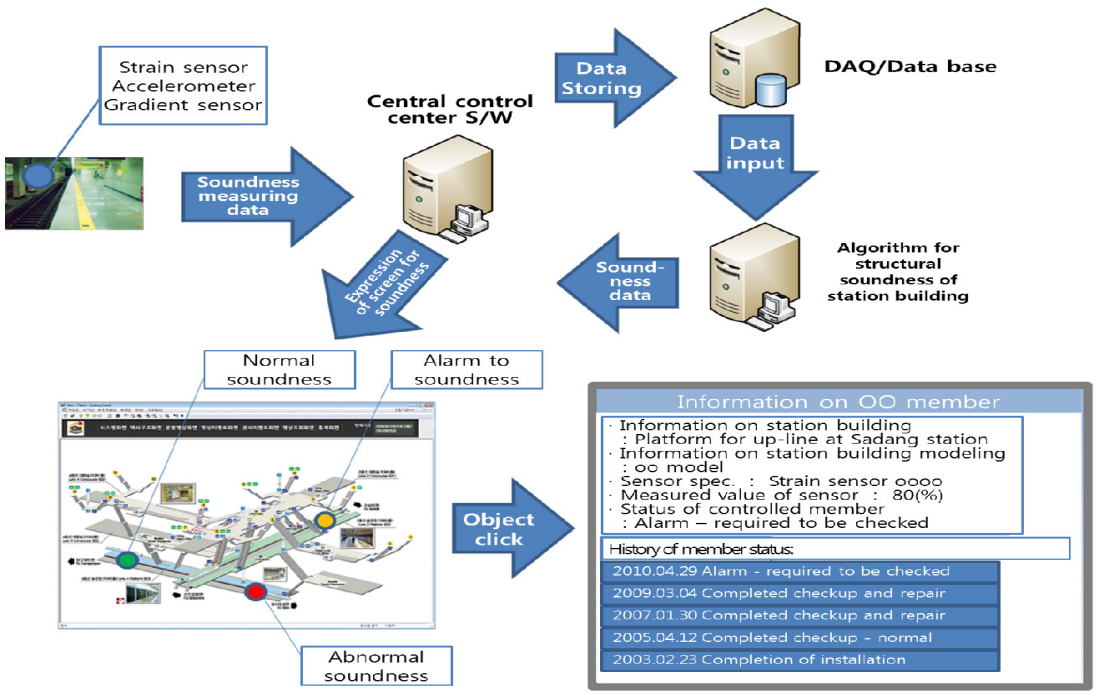

Figure 5: $\quad$ Station building structural soundness surveillance scenario.

\subsection{Fire detection function}

Fire detection function is the function which makes the surveillance operator recognize information on whether any fire was occurred, information on the area occurred on the basis of image and sensor data to be transmitted from the camera and fire sensor, and that spreads the fire occurrence situation to the central control center. 

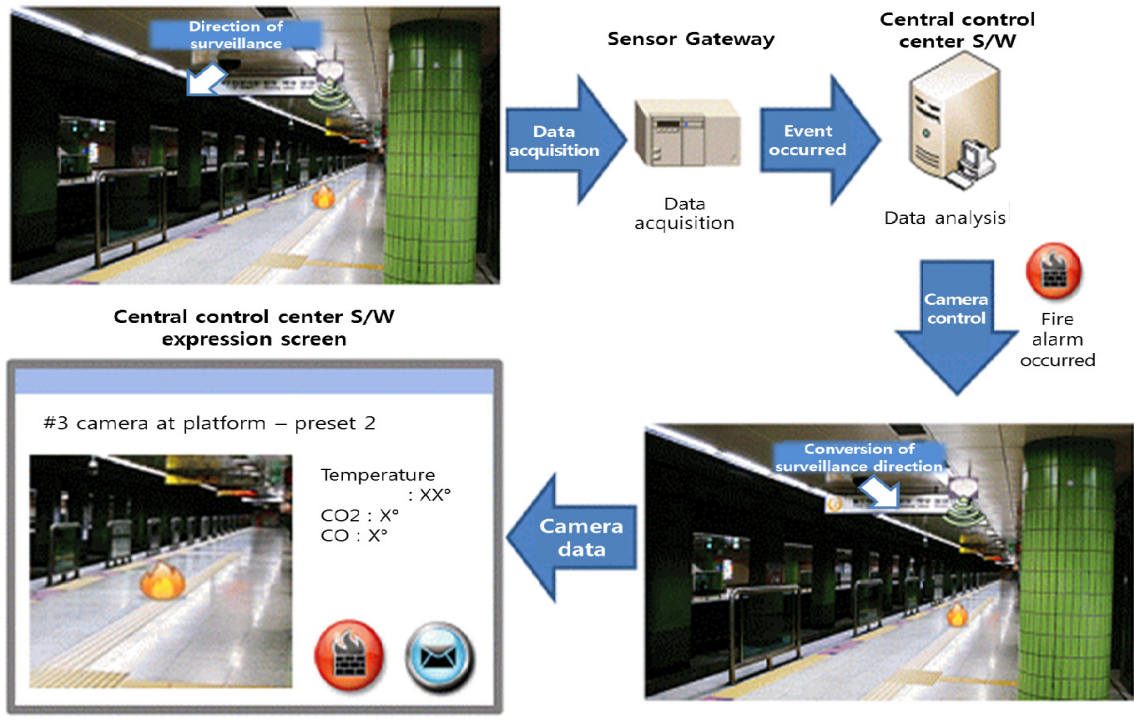

Figure 6: $\quad$ Fire detection scenario.

\subsection{Event occurrence function through complex data analysis}

The central control center S/W of intelligent surveillance system has the function to make the event occurred through complex data analysis by the setting of the person in charge of surveillance. The most important thing in the fire situation is the rapid response and safe evacuation of passengers.
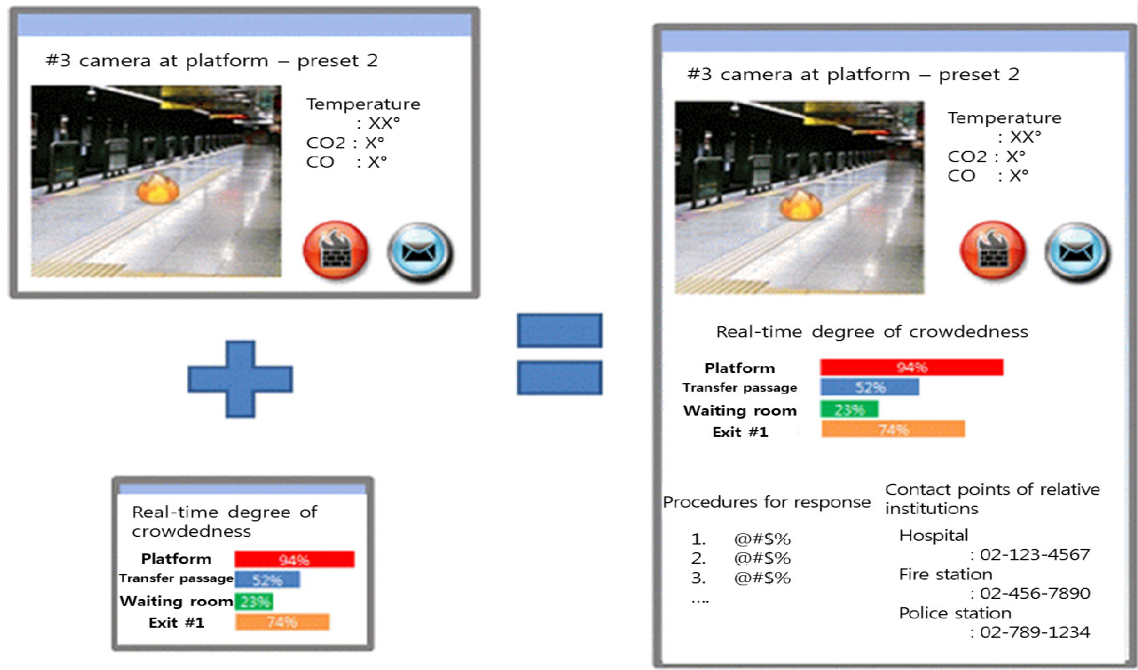

Figure 7: Event occurrence scenario through complex data analysis. 


\section{Performance test on the unit module of intelligent surveillance system}

To test the performance by unit module of intelligent surveillance system, a trial examination was progressed at the Suseo station, Suseo vehicle depot and Chungmooro station of Seoul Metro. Development is under progress now, and the test for two functions among total of 7 system requirements was progressed as below, and the test for remaining requirements will be carried out also.

\subsection{Performance test for the Enter module of intelligent surveillance camera}

1) Test item: detection of object which was abandoned and removed

2) Functions in relation to R\&D: invasion detection function in the restricted area, designated/ crime-ridden area surveillance function

3) Test requirements

- It does not operate properly when the object size is in excess due to the serious blocking or when the object is in lack of detection from the remote distance. It must be existed within the area of object size 1-10 at the inside of system.

- Experiment must be progressed at the place where the environmental change which can affect object detection is not made seriously. For example, it is not operated normally at the place where elements such as rapid change of illumination or snow, rain, cloud, fog, etc. that change images frequently are existed.

- Interested area shall have the width which is wider than average size of object in the environment required to be detected.

4) Test procedures

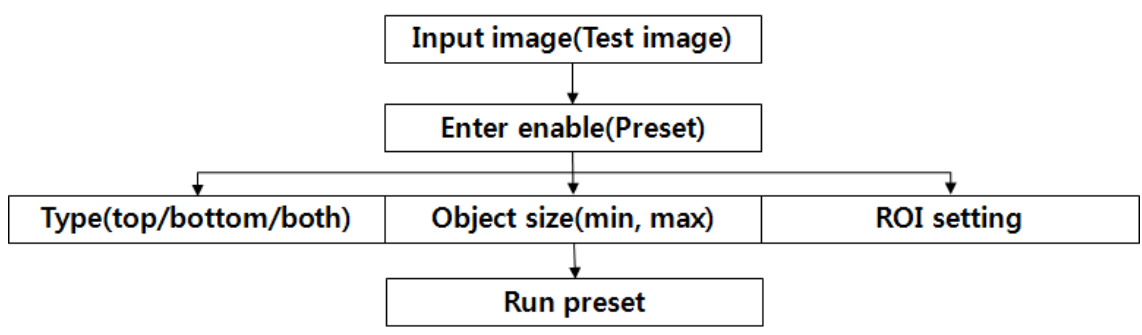

5) Result of experiment

- Four times of elevator boarding events occurred during the progress of photographing for three minutes.

- Out of detection on four persons, no-detection or wrong detection did not occur and the function was performed normally.

- Although the blob of detected object was split since the illumination around the elevator was lower than that of another area, there was no hindrance in the performance. 
- As a result of performing several more times under the same condition by taking pictures of the elevator at the interval of about three minutes, nodetection or wrong detection did not occur (test time twenty minutes)

6) Conclusion

- No bug occurred as a result of a test on performance of Enter based on our own validation.

- In the process of detection on object blob, it is judged that additional considerations on image enhancement are necessary in order to minimize the effect of illumination.

- Enter function can display its maximum performance since environmental changes would be less if it is installed at the restricted area, elevator or in front of the station affairs office mainly used by specific persons (manager, the old and the weak, and the disabled) or at the area where the appearance frequency of persons is not high.

- Appropriate place when installed: in front of the elevator, restricted area (communication room, air conditioning room), wheelchair lift (event will be transmitted to the station affairs office by sensing it automatically if anyone comes into the blind spot), near to the station affairs office, etc.

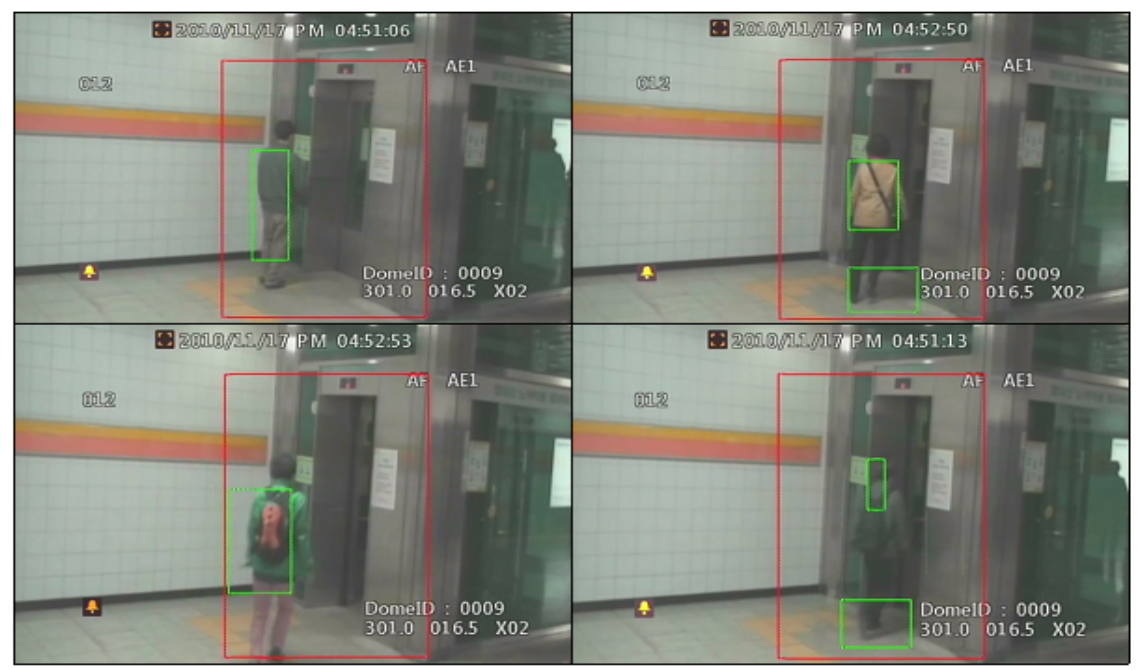

\subsection{Performance test on the cross module of intelligent surveillance camera}

1) Test item: detection function.

2) Function in relation to R\&D: Invasion detection function in the restricted area.

3) Test requirements

- The object must exist within the area of object size 1-10 at the inside of the system. That is, it does not operate properly when the object size is in excess or when the object is lacking detection from the remote distance. 
- Experiment must progress at the place where the environmental change, which can affect object detection, is not made seriously. For example, it is not operated normally at the place where elements such as rapid change of illumination or snow, rain, cloud, fog, etc. that change images frequently exist.

- Necessary direction or two-way shall be set accurately.

4) Test procedures

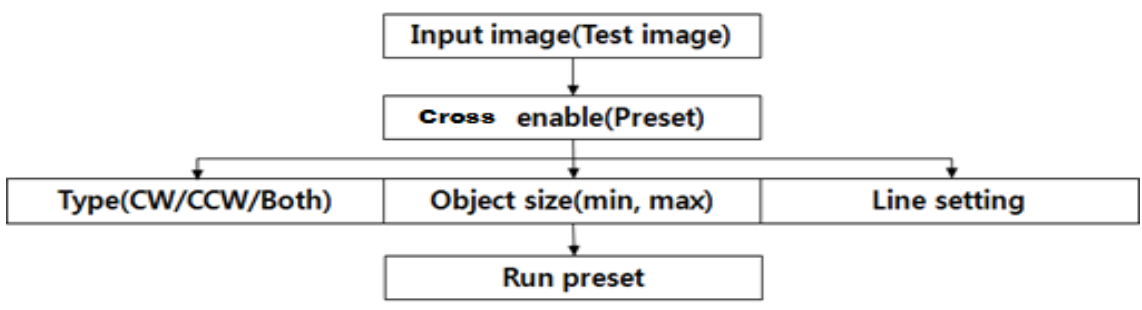

5) Result of experiment

- Photographs were taken for about five minutes at one time, and two images at the top end were detected from two-way, and two images at the bottom end were detected from the direction toward the outside.

- No-detection or wrong detection did not occur and the function was performed normally in accordance with the direction.

- It catches any bug for the illegal pass at the ticket gate accurately.

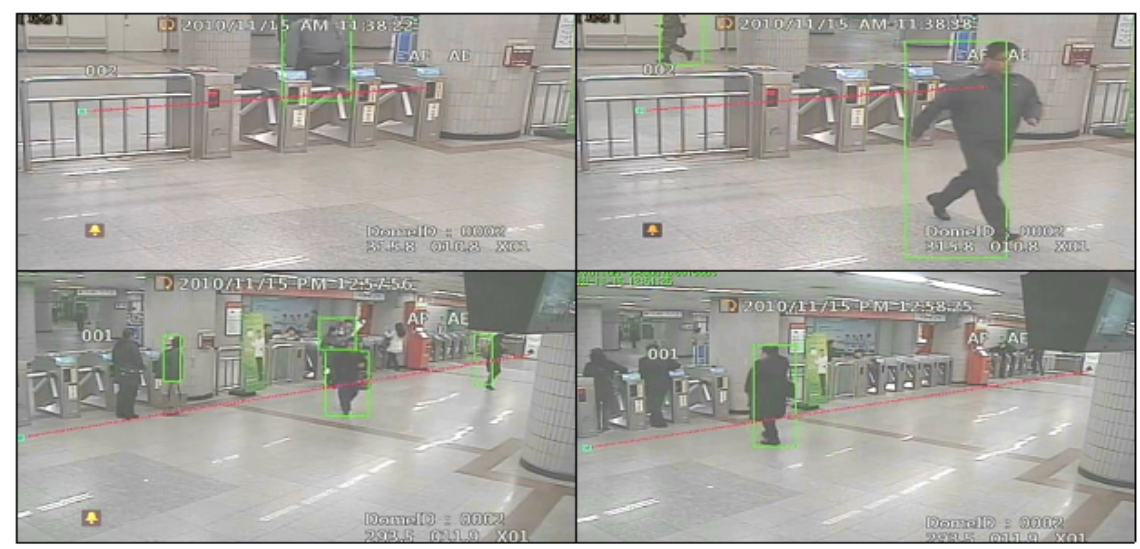

6) Conclusion

- As a result of a test on performance of Cross according to its own validation, no bug occurred.

- In accordance with the location of the line, the function to detect illegal invasion was also performed properly.

- Cross function can grasp the effect of surveillance properly if it is installed at the area where the appearance frequency of a person is somewhat high, such as the ticket gate and the platform without screen door, etc.

- It is judged that the effect of surveillance will be much bigger if the counting 
function is combined with it.

- An appropriate place when installed: the place where the movement frequency of people is appropriately high, such as in front of a ticket gate, escalator, etc.

\subsection{Performance test on the Abandon and Removal module of intelligent surveillance camera}

1) Test item: detection of object which was abandoned and removed.

2) Function in relation to $R \& D$ : designated/crime-ridden area surveillance function.

3) Test requirements

- The camera shall be located at a height of more than $2 \mathrm{~m}$ above ground.

- The camera must be able to acquire images which are not swaying.

- The image input from the camera must not be distorted from the noise occurred from problems in hardware or environmental factors.

4) Test procedures

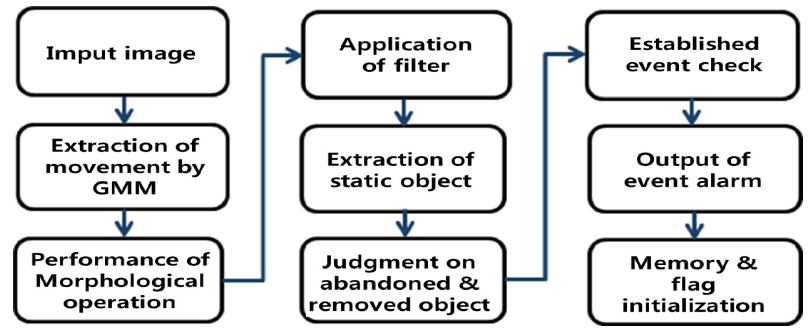

5) Performance judgment criteria

(rate of perception was measured after testing more than ten times)

90\%-100\%: Best/80\%-90\%: Good/70\%-80\%: Fair/Below 70\%: Poor

6) Result of test

- The performance is distinguished as "Fair" since the rate of perception was $81 \%$.

- The event might not occur, but the bug detected as if an event was detected; about $10 \%$.

- The cases where the abandoned and removed object was distinguished mistakenly occurred twice.

- As of April 2011, we analyzed problems and modified our program design by dividing cases which were distinguished mistakenly into the wrong judgment and wrong detection to enhance the rate of perception. As a result of a retest, performance was enhanced by around $85 \%$.

\section{Conclusion}

A performance test on some of the functional modules were carried out at the Suseo station of Seoul Metro, and the remaining functional requirements are planned to be tested by module also. Integrated functional test of intelligent 
surveillance system will be performed after completion of test on the functional modules, and the corresponding configuration chart is as below.

We installed hardware equipment to install the prototype at the Chungmooro station building of Seoul Metro to test unit modules of central control center $\mathrm{S} / \mathrm{W}$, and the functional test, grasping of problem, analysis on additional functional requirements and additional functional definition are under progress by installing the central control center S/W and digital image storing device S/W implemented to date at the installed equipment, and the synthetic linkage test will be progressed through installing the redundancy equipment of general station building and management branch office, and the equipment at the central control center.

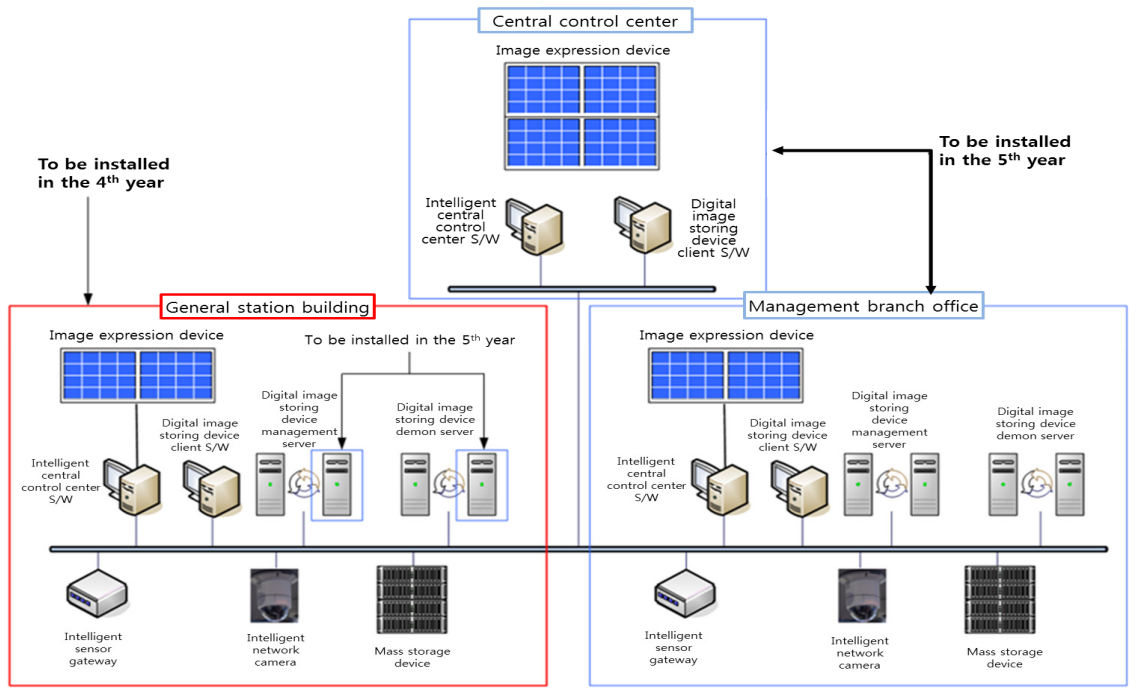

Figure 8: Entire test configuration chart

By applying this system, it is possible to build a standardized urban transit surveillance system, and it is possible to apply it immediately through standardized system even though any product whose event detection ability is enhanced would be developed in the future through gradual development of surveillance system. In addition, the standardization of urban transit surveillance system can be built gradually by developing not only station buildings but also central control center $\mathrm{S} / \mathrm{W}$ by dividing them. As the practical product reflecting properties of the field to the maximum by making it put to practical use and commercialized as product by reflecting maximum requirements of field operator, it will be exported as the product applicable even in the global market immediately. We are planning to foster it as the future industry by developing its original technology through continuous investment. Since it is the system where various information processing technologies such as the image surveillance and network data processing, sensor network, database-related technology, etc. are 
grafted, we have a plan to utilize it actively taking into consideration that the ripple effect on other industries will be great.

\section{References}

[1] Cheolmin Choi, Sooyeong Kwak, Jungho Ahn, Hyeran Byun, “Occluded Object Tracking in Moving Camera Environment”, Korea Computer Congress, vol. 33, no. 1(B), 2006.

[2] Byeoung-su Kim, Gwang-Gook Lee, Ja-Young Yoon, Jae-Jun Kim, WhoiYul Kim, "An Effective Shadow Elimination Method Using Adaptive Parameters Update”, IEEK, vol. 45, SP no. 3, May, 2008

[3] Hyun-Koong Kang, Kwang-hyoung Lee, "Realtime Object Extraction and Tracking System for Moving Object Monitoring”, The Korean Society of Computer and Information, vol. 10, no. 2, pp. 59-68, 2005

[4] Feng Liu, Qingshan Liu and Hanqing Lu, "Robust color-based tracking," IEEE, proceedings of the third international conference on image and graphics (ICIG'04), 2004.

[5] Takuma Funahasahi, Masafumi Tominaga, Takayuki Fujiwara and Hiroyasu Koshimizu, "Hierarchical face tracking by using PTZ camera," IEEE, Proceedings of the Sixth IEEE International Conference on Automatic Face and Gesture Recognition (FGR'04), 2004.

[6] Bo Zhang, Weifeng Tian and Zhihua Jin, "Joint tracking algorithm using particle filter and mean shift with target model updating," Chinese Optics Letters, vol. 4, no. 10, October, 2006. 